\title{
PReS-FINAL-2019: Tuberculosis infection observed in patients receiving biological DMARDs for treatment of juvenile idiopathic arthritis
}

\author{
A Sarychev ${ }^{1 *}$, L Shovkun ${ }^{2}$ \\ From 20th Pediatric Rheumatology European Society (PReS) Congress \\ Ljubljana, Slovenia. 25-29 September 2013
}

\section{Introduction}

The use of immunosuppressant drugs and also biological DMARDs in the treatment of juvenile idiopathic arthritis (JIA) may weaken patient's immunity and raise the risk of serious infections, including tuberculosis (TB).

\section{Objectives}

To assess the incidence rate of different forms of TB infection in patients receiving biological treatment of JIA.

\section{Methods}

Thirty four children, aged from 3 to 17 years, were observed prospectively receiving biological treatment for JIA. Mean disease duration was 5,29 $( \pm 4,24)$ yrs. Initial screening for TB included PPD test and chest CT.

\section{Results}

Fourteen of 34 patients $(41,2 \%)$ received toziliaumab (TOZ), 6 (17,6\%) - abatacept (ABA), 4 (11,8\%) - etanercept (ETN), $5(14,7 \%)$ - adalimumab (ADA) and $5(14,7 \%)$ infliximab (IFL). Mean treatment duration with biologics was 16,3 $( \pm 10,47)$, from 3 to 46 months. Different types of TB infection were diagnosed in $6(17,6 \%)$ patients while receiving biological DMARDs. Three of them received treatment for systemic JIA, 1 - for polyarticular JIA, 1 juvenile ankylosing spondylitis and 1- JIA with concomitant uveitis. Different clinical types of TB infection were observed in these 6 patients: one patient receiving TOZ developed infiltrative pulmonary TB with dissemination, two patients ( 1 on TOZ and 1 on ADA) had primary TB complex and three (on IFL, ADA, ETN) were diagnosed with latent $\mathrm{TB}$ infection. The common feature $\mathrm{TB}$ infection in all patients receiving biologics was the mildness of clinical manifestation of the disease.

In addition 3 cases of primary TB complex were observed in patients with systemic JIA before initiation of biological DMARD and required prior specific TB therapy.

\section{Conclusion}

Children with systemic JIA may be considered to have the higher risk of different types of TB infection. Treatment with biological DMARDs (especially with TNF- $\alpha$ and IL-6 inhibitors) also may contribute to TB infection. Regular TB screening (at least once per 6 months) is required to the scarcity of clinical manifestation of the infection.

\section{Disclosure of interest}

None declared.

\section{Authors' details}

${ }^{1}$ Pediatrics, Russian Federation. ${ }^{2}$ Rostov State Medical University, ROSTOV-NADONU, Russian Federation.

Published: 5 December 2013

doi:10.1186/1546-0096-11-S2-P32

Cite this article as: Sarychev and Shovkun: PReS-FINAL-2019:

Tuberculosis infection observed in patients receiving biological

DMARDs for treatment of juvenile idiopathic arthritis. Pediatric

Rheumatology 2013 11(Suppl 2):P32.

${ }^{1}$ Pediatrics, Russian Federation

Full list of author information is available at the end of the article 